\title{
Cateteres umbilicais em recém-nascidos: indicações, complicações e diagnóstico por imagem
}

\author{
Umbilical catheters in newborns: indications, complications and imaging diagnosis
}

\author{
Ricardo Yoshio Zanetti Kido ${ }^{1,4} \bowtie$, Beatriz Regina Alvares ${ }^{2,4}$, Maria Aparecida Marques dos Santos Mezzacappa ${ }^{3,4}$ \\ ${ }^{1}$ Médico Residente do Departamento de Radiologia. \\ 2 Professora Doutora do Departamento de Radiologia. \\ 3 Professora Doutora do Departamento de Pediatria. \\ ${ }^{4}$ Faculdade de Ciências Médicas da Universidade Estadual de Campinas (FCM-Unicamp), Campinas, SP, Brasil.
}

\section{RESUMO}

Objetivos: Revisar os últimos dados de literatura acerca do cateterismo umbilical quanto a indicações, complicações e diagnóstico de sua localização através de exames de imagem.

Métodos: Foram pesquisados artigos nas bases de dados PubMed, SciELO e LILACS, através dos seguintes descritores, assim como dos termos correspondentes em inglês: cateteres umbilicais; exame radiológico; ultrassonografia; recém-nascido; e cuidados intensivos neonatais. Foram incluídos também livros texto clássicos em literatura neonatal.

Resultados: Foram selecionados 51 artigos, quatro livros texto e um manual versando sobre cateteres umbilicais venosos e arteriais em recém-nascidos. Revisamos indicações, novas técnicas de inserção, complicações associadas, fatores de risco e diagnóstico por imagem, com ênfase nas referências anatômicas utilizadas para o diagnóstico da posição da extremidade do cateter.

Conclusões: $\mathrm{O}$ cateterismo umbilical representa um importante acesso à corrente sanguínea do recém-nascido e o diagnóstico por imagem desempenha um papel fundamental na avaliação correta do seu posicionamento, possibilitando, em caso de mau posicionamento, a conduta imediata para prevenção de complicações.

DESCRITORES: cateteres; radiografia; ultrassonografia; recém-nascido; cuidados intensivos.

\section{ABSTRACT}

Aims: To review the latest literature on umbilical catheterization including its indications, complications, and diagnostic imaging for verification of catheter position.

Methods: The PubMed, SciELO, and LILACS databases were searched using the following keywords and the corresponding terms in Portuguese: umbilical catheters; radiological examination; ultrasonography; newborn; and neonatal intensive care. Classic textbooks on neonatology were also reviewed.

Results: A total of 51 articles, four classic textbooks, and one manual dealing with venous and arterial umbilical catheterization in neonates were selected. We reviewed the indications, new insertion techniques, complications, risk factors and, diagnostic imaging, with emphasis on the anatomic landmarks used for determination of catheter tip position.

Conclusions: Umbilical catheterization is an important access to the bloodstream of the newborn, and diagnostic imaging plays a key role in the assessment of catheter tip position, thus enabling immediate management and preventing complications in cases of misplacement.

KEY WORDS: catheters; radiography; ultrasonography; infant, newborn; intensive care.

Recebido: novembro, 2014 


\section{INTRODUÇÃO}

O uso do cateterismo umbilical venoso e arterial em recém-nascidos representa uma prática rotineira em unidade de terapia intensiva (UTI) neonatal, com finalidade de infundir líquidos e medicações, coletar amostras de sangue, monitorar pressão arterial e pressão venosa central e realizar exsanguineotransfusões [1-5], sendo uma via prontamente acessível durante a reanimação neonatal. Aproximadamente após três meses do nascimento os vasos umbilicais transformam-se em estruturas ligamentares, mas nas primeiras horas de vida ainda podem ser utilizados como acesso [6].

A posição incorreta da extremidade do cateter umbilical associa-se a múltiplas e graves complicações de ordem vascular, cardíaca e infecciosa, bem como a lesões relacionadas com o procedimento de inserção [7-12]. Assim, o exame radiológico de tórax e abdome deve ser realizado logo após a realização do cateterismo, para confirmar a localização do cateter e, se necessário, orientar as condutas de recolocação ou remoção do mesmo [13-15].

O objetivo deste estudo foi revisar os dados de literatura acerca do cateterismo umbilical de recém-nascidos, quanto a indicações, complicações e diagnóstico da localização do cateter através de exames de imagem, além de demonstrar, com imagens de radiografias simples de tórax e abdome, os posicionamentos adequados e inadequados.

\section{MÉTODOS}

Entre julho de 2013 e agosto de 2014 realizou-se um levantamento bibliográfico através das bases de dados Medline/PubMed, LILACS e SciELO, acessadas por intermédio da página eletrônica da Biblioteca Regional de Medicina (BIREME), utilizando-se as palavras-chave umbilical catethers; radiography; ultrassonography; newborn; neonatal intensive care e seus equivalentes em português. Outras fontes utilizadas foram livros textos clássicos em radiologia e cuidados intensivos neonatais. Foi dada preferência a trabalhos recentes, especialmente publicados na última década, porém alguns artigos clássicos foram incluídos devido ao pioneirismo de suas informações. Exemplificamos os achados desta revisão com radiografias de recém-nascidos internados em Unidade de Terapia Intensiva (UTI) Neonatal e submetidos a cateterismo umbilical arterial e/ou venoso. As imagens apresentadas foram obtidas de neonatos internados na UTI Neonatal do Centro de Atenção Integral à Saúde da Mulher (CAISM-UNICAMP) e aprovadas pela diretoria clínica da instituição para esta publicação, após ter sido removida a identificação dos pacientes.

\section{RESULTADOS DA SELEÇÃO}

Estavam disponíveis 1451 artigos nas bases de dados eletrônicas a partir das palavras chave, versando sobre os inúmeros aspectos do cateterismo umbilical, sendo selecionados os artigos após a análise de títulos e resumos. Ao final da revisão, foram incluídos 51 artigos, quatro livros texto e um manual de condutas do Ministério da Saúde versando sobre o posicionamento de cateteres umbilicais venosos e arteriais em recémnascidos, complicações associadas, fatores de risco e diagnóstico por imagem.

\section{CONTEÚDO DA REVISÃO}

\section{Cateterismo umbilical}

O cateter umbilical arterial é introduzido no recémnascido em uma das duas artérias umbilicais, passando pelas artérias ilíaca interna, ilíaca comum e aorta. A ponta do cateter umbilical arterial deve ficar situada na aorta e distante do orifício de qualquer artéria de menor calibre, visando a evitar a oclusão desses vasos e a injeção direta de soluções hipertônicas ou com pH elevado em sua luz. As duas localizações que atendem a esse critério são a aorta abdominal, logo acima da bifurcação das artérias ilíacas (localização baixa), e a aorta torácica, entre o ducto arterioso e o tronco celíaco (localização alta). O cateter umbilical venoso, após sua introdução, cursa pela veia umbilical, ramo esquerdo da veia porta e ducto venoso, entrando na veia cava inferior, onde deve ficar locado, próximo ao átrio direito $[12,13,16,17]$.

Um dos grandes desafios no posicionamento adequado dos cateteres umbilicais é a determinação da extensão a ser introduzida, possibilitando um posicionamento sem risco de complicações. Mesmo obedecendo a técnicas padronizadas, é frequente a observação de cateteres não adequadamente locados, de modo que novas técnicas de inserção vêm sendo desenvolvidas na tentativa de minimizar o mau posicionamento [18]. Várias técnicas, que tomam como base o peso do recém -nascido ou distâncias corporais como a distância ombro umbigo, têm sido utilizadas [19-21]. O estudo de Dunn [21] foi realizado em cadáveres e desenvolveu um nomograma que permite estimar a distância a ser introduzida medindose o comprimento do ombro até a cicatriz umbilical. 
Como a casuística de recém-nascidos com menos de $1500 \mathrm{~g}$ nesses estudos foi reduzida, as estimativas desses nomogramas podem não ser muito adequadas para essa faixa ponderal, que nos dias atuais é a que mais se utiliza do cateterismo umbilical. Cateteres umbilicais são indicados para recém-nascidos instáveis, que precisam de acesso venoso de urgência, monitorização de gases sanguíneos frequentes, monitorização da pressão arterial e da pressão venosa central, ou exsanguineotransfusão. Após cinco a sete dias essa via é substituída por cateteres centrais de inserção periférica (PICC). Wright et al. [19] demonstraram, em um ensaio randomizado controlado, realizado em 74 recém-nascidos de muito baixo peso, que houve diferença estatística entre o nomograma de Dunn e uma nova fórmula proposta pelos autores: comprimento do cateter umbilical arterial $=4 \times$ peso $\mathrm{em} \mathrm{kg}+7$. Verheij et al. [4], em um estudo com 139 recém-nascidos, demonstraram que a fórmula de Shukla, $(3 \times$ peso de nascimento em $\mathrm{kg}+9) / 2+1 \mathrm{~cm}$, apresenta maiores taxas de hiperinserção do cateter em comparação com a seguinte fórmula revisada: $(3 \times$ peso de nascimento em $\mathrm{kg}+9) / 2$, cuja diferença básica em relação à fórmula original é a não inclusão de $1 \mathrm{~cm}$ ao final da equação. Tais estudos demonstram uma constante preocupação e tentativas de melhorias nas técnicas de inserção do cateter umbilical.

\section{Complicações associadas ao mau posicionamento dos cateteres umbilicais}

A posição incorreta da extremidade do cateter umbilical e um tempo grande de permanência do mesmo em localização inadequada podem ocasionar graves complicações [2]. Problemas de ordem vascular podem ser decorrentes de espasmo, laceração, hemorragia, fenômenos tromboembólicos e isquêmicos. $\mathrm{O}$ espasmo vascular ocorre de maneira aguda, sendo mais frequentemente relacionado aos cateteres arteriais, minutos a horas após sua inserção. A suspeita de espasmo vascular ocorre na presença de sinais isquêmicos distais progressivos, como palidez de um membro, ausência de pulso e gangrena em casos avançados [1,6,7,23]. Cateteres umbilicais arteriais em posição baixa (T12-L3) podem estar associados à redução de fluxo nas artérias renais de recém-nascidos pré-termo, podendo reduzir o débito urinário [6,24]. $O$ tromboembolismo da artéria renal é geralmente iatrogênico, relacionado ao cateterismo da artéria umbilical, representando a principal causa de hipertensão renovascular em recémnascidos [22].
Os eventos tromboembólicos ocorrem nos sítios venoso e arterial e decorrem da lesão endotelial causada por um corpo estranho com superfície trombogênica $[23,25]$, principalmente em recémnascidos com outros fatores de risco para trombogênese, como policitemia neonatal, pré-eclâmpsia materna, prematuridade, desidratação e septicemia, entre outros [26,27]. Com relação ao tempo de cateterização, os cateteres umbilicais arteriais apresentam risco de trombose diretamente proporcional ao seu tempo de permanência, sendo incomum essa complicação nos primeiros cinco dias de inserção [26,28]. Estudos referem que o cateter venoso apresenta maior risco de causar trombose do que o arterial $[26,29,30]$. A presença de cateter umbilical por mais de seis dias predispõe a trombose portal, sendo que a maioria evolui com recanalização portal sem sequelas clínicas [22]. É possível também que ocorra a perfuração da veia porta pelo cateter, levando a hemoperitôneo [31].

As complicações decorrentes da inadequada localização intracardíaca do cateter umbilical são graves e potencialmente fatais. Nessa localização, seja por movimentação do recém-nascido, ciclo cardíaco ou respiração, a extremidade do cateter faz contato com o endocárdio e, quanto mais perpendicular ela estiver junto à parede cardíaca, maior a chance de trauma direto e erosão, potencializada pela infusão de substâncias hiperosmolares que ocasionam lesão osmótica no endotélio, levando a perfuração atrial, tamponamento cardíaco e arritmias [31-33]. Os cateteres intracardíacos podem induzir mecanicamente estímulos elétricos atriais, podendo ser um gatilho para o início de um flutter atrial ou uma taquicardia supraventricular na presença de um feixe anômalo atrioventricular, sendo estas as arritmias mais frequentes nesse tipo de situação [34].

As complicações sépticas têm taxa de incidência de 5 a $57 \%$ entre todos os cateterismos umbilicais [6] e estão relacionadas à infecção da corrente sanguínea, uma vez que o cateter pode atuar como um acesso direto de microorganismos à circulação [35]. ButlerO'Hara et al. [5], em um estudo retrospectivo com 196 recém-nascidos, verificaram que dentre todos os tipos de cateteres vasculares, os umbilicais foram aqueles com maiores taxas de infecção, sendo estas diretamente proporcionais ao tempo de permanência do cateter. Naquele estudo também foi constatado que a prematuridade e o baixo peso ao nascer representaram fatores relevantes para maior tempo de internação dos recém-nascidos, atuando de maneira indireta como fator de risco para infecção relacionada 
ao cateter umbilical [5]. Outro estudo demonstrou que a utilização frequente de cateteres umbilicais aumentou significativamente a incidência de abscessos hepáticos [35]. Vale ressaltar que, segundo o Ministério da Saúde, cateteres duplo lúmen podem ser utilizados no cateterismo venoso apenas em situações críticas, devido ao maior risco de contaminação [1].

Outros estudos identificaram como fatores de risco para infecção em recém-nascidos com cateteres umbilicais a baixa idade gestacional, o maior tempo de permanência e o alto grau de manipulação do cateter e o seu uso para nutrição parenteral [19]. Em todos os estudos, incluindo os demais tipos de cateteres centrais, o principal agente infeccioso foi o Staphylococcus coagulase negativo [36, 37]. A manutenção do cateter umblilical através de solução heparinizada deve ser evitada por se associar à infecção fúngica, devendo ser realizada apenas por solução salina, com os devidos cuidados com a infusão de sódio no recém-nascido [1].

Outras complicações menos comuns devem ser lembradas, como lesão vascular hepática com extravasamento de nutrição parenteral intraparenquimatosa $[22,38]$, perfuração iatrogênica de divertículo de Meckel [39] e fístula venobiliar [40]. Em alguns casos pode ocorrer a injeção de ar pelo sistema portal durante a inserção de cateter umbilical venoso, achado que pode ser inadvertidamente confundido com enterocolite necrosante, devendo nesses casos ser realizada a correlação com dados clínicos do recémnascido e outros achados radiológicos patognomônicos dessa doença, especialmente a presença de pneumatose intestinal [41].

\section{Exame ultrassonográfico}

O exame ultrassonográfico é um método diagnóstico que pode ser utilizado para a localização dos cateteres umbilicais. Apresenta como vantagens não usar radiação ionizante e não precisar de referências anatômicas para identificar a localização dos cateteres, já que as estruturas vasculares são visualizadas durante a realização do exame, podendo fornecer informações mais precisas que o exame radiológico [42]. Além disso, a ultrassonografia não precisa utilizar marcos anatômicos que podem variar entre os recém-nascidos, como a altura da bifurcação da aorta, que ocorre ao nível de L4 em apenas $60 \%$ dos casos [43].

Michel et al. [44] compararam ambos os métodos de imagem e demonstraram que a sensibilidade e a especificidade para determinar a trajetória do cateter foram respectivamente de $96,4 \%$ e $93,9 \%$ na ultrassonografia e de $92,8 \%$ e $78,8 \%$ no exame radiológico. Para estimar a localização da extremidade do cateter, a sensibilidade e a especificidade obtidas foram respectivamente de $93,3 \%$ e $95,6 \%$ na ultrassonografia e de $66,7 \%$ e $63,0 \%$ no exame radiológico. Os autores concluíram que ambos os métodos foram confiáveis para determinar a trajetória do cateter (central ou não), mas a ultrassonografia foi superior em determinar a posição da ponta do cateter [44].

Simanovsky et al. [45], em um estudo com 75 recém-nascidos, não constataram diferença estatística entre radiografia e ultrassonografia na determinação do posicionamento do cateter venoso umbilical, havendo boa correlação entre ambos os métodos. No mesmo estudo, a ultrassonografia possibilitou demonstrar a exata localização da ponta do cateter em $97 \%$ dos pacientes, permitindo a rápida detecção em casos de mau posicionamento, evitando complicações graves [45].

O exame radiológico apresenta como desvantagem o uso de radiação ionizante, devendo, portanto, ser usado de forma criteriosa. Greenberg et al. [14] constataram que $56 \%$ dos cateteres umbilicais avaliados através de exames radiológicos tiveram que ser reposicionados devido à localização inadequada, sendo necessária a realização de nova radiografia após esse procedimento, submetendo o recém-nascido a uma carga de radiação adicional.

Apesar de o exame ultrassonográfico apresentar vantagens em relação ao exame radiológico, como a não utilização da radiação ionizante e poder determinar a localização em tempo real dos cateteres umbilicais, ele apresenta como desvantagem a menor disponibilidade de profissionais treinados para a sua realização, fazendo com que o exame radiológico de tórax e abdome continue representando o procedimento mais usado e disponível até o presente momento para avaliar a correta localização dos cateteres umbilicais, podendo ser realizado na própria UTI neonatal, com aparelho portátil $[44,46]$.

\section{Exame radiológico}

O exame radiológico simples de tórax e abdome, realizado com o recém-nascido em decúbito dorsal com raios verticais, possibilita identificar a exata localização dos cateteres umbilicais no interior das estruturas vasculares, através da sua trajetória e de referências anatômicas previamente estabelecidas $[44,47,48]$. No início da sua inserção, o cateter umbilical arterial apresenta uma pequena curvatura, coincidindo com a sua entrada na artéria ilíaca interna 
e aorta abdominal, possibilitando a sua diferenciação com o cateter umbilical venoso, que apresenta uma trajetória retilínea, sendo visualizado à direita dos corpos vertebrais [12].

Dados de literatura demonstram variabilidade em relação às referências anatômicas que orientam o posicionamento correto, tanto do cateter umbilical arterial quanto do cateter umbilical venoso. Dessa forma, são citados como referências anatômicas para a localização correta do cateter umbilical arterial alto, com localização na aorta torácica, os corpos vertebrais de T6 a T10 [1,6,16,22,40-50] (Figura 1). Para a localização baixa do cateter umbilical arterial, situado na aorta abdominal, logo acima das artérias ilíacas, são citados como referência os corpos vertebrais de L3 a L4 [1,6,12,16,22,23,31,49] (Figura 2). Devem-se evitar localizações do cateter umbilical arterial na aorta abdominal entre os corpos vertebrais

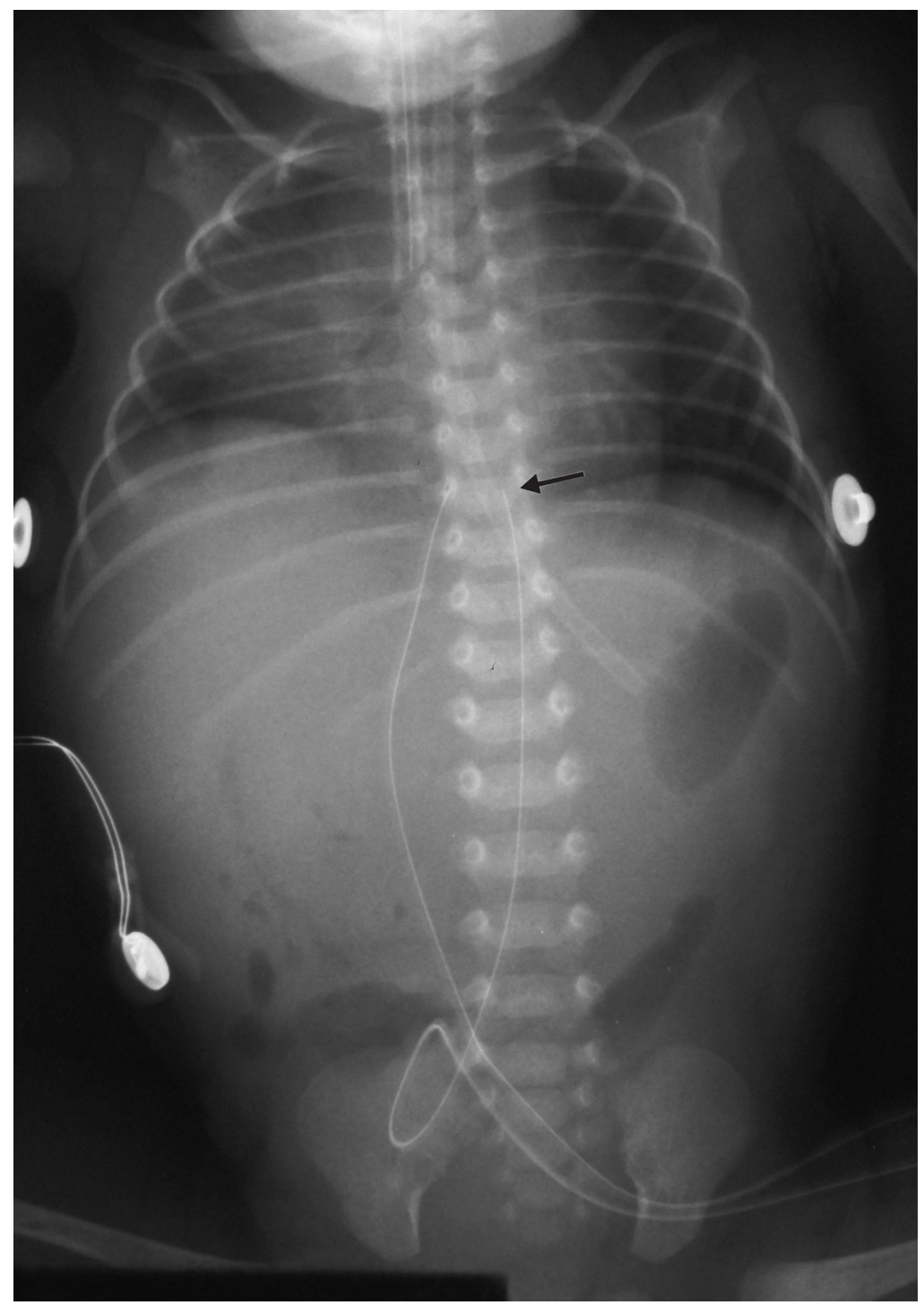

de T11 a L3, devido aos locais do tronco celíaco (T11-T12), artéria mesentérica superior (T12-L1), artérias renais (T12-L3) e artéria mesentérica inferior (L2) $[16,31]$.

$\mathrm{O}$ posicionamento mais adequado para a extremidade do cateter umbilical venoso é na veia cava inferior, próximo à sua entrada no átrio direito $[51,52]$, usando-se como referências anatômicas os corpos vertebrais de T8 a T9 [53] (Figura 3). Radiologicamente, o cateter apresenta uma trajetória retilínea, à direita da coluna torácica, evidenciando algumas vezes uma discreta curvatura ao nível de T10-L1, correspondendo à sua passagem da veia umbilical para a veia cava inferior através do ducto venoso [54]. Frequentemente os cateteres umbilicais podem ser inseridos inadvertidamente nos ramos da veia porta e/ou da veia esplênica, podendo essas localizações ser detectadas radiologicamente por sua visualização ao nível dos corpos vertebrais de T10 a T12 e apresentando a sua extremidade encurvada para a direita (topografia do ramo direito da veia porta) ou para a esquerda (topografia do ramo esquerdo da veia porta ou da veia esplênica), havendo necessidade de reposicionamento [11,55] (Figura 4). O posicionamento correto do cateter venoso umbilical na primeira tentativa varia de $50 \%$ a $90 \%[12,56]$. Considerando os corpos vertebrais de T8 a T9 como referências anatômicas para a localização do cateter umbilical venoso na veia cava inferior, caso a extremidade do cateter esteja localizada entre os corpos vertebrais de $\mathrm{T} 6$ a T7, existe grande probabilidade de ele estar no interior

Figura 1. Radiografia de tórax e abdome de recém-nascido com 24 horas de vida, idade gestacional de 39 semanas e peso ao nascer de 2620 g, apresentando insuficiência respiratória e submetido a cateterismo umbilical venoso e arterial. As extremidades distais de ambos os cateteres são visíveis sobre o corpo vertebral de T9, estando bem locados (cateter venoso à direita, em topografia de veia cava inferior, e cateter arterial à esquerda, em localização anatômica da aorta torácica). Observa-se também presença de cânula endotraqueal com extremidade visível entre os corpos vertebrais de T4-T5, estando abaixo da localização considerada adequada (corpo vertebral de T1), devendo ser reposicionada. A sonda gástrica é visível na bolha de ar do estômago.

O posicionamento do recém-nascido está adequado para a finalidade do exame radiológico. 


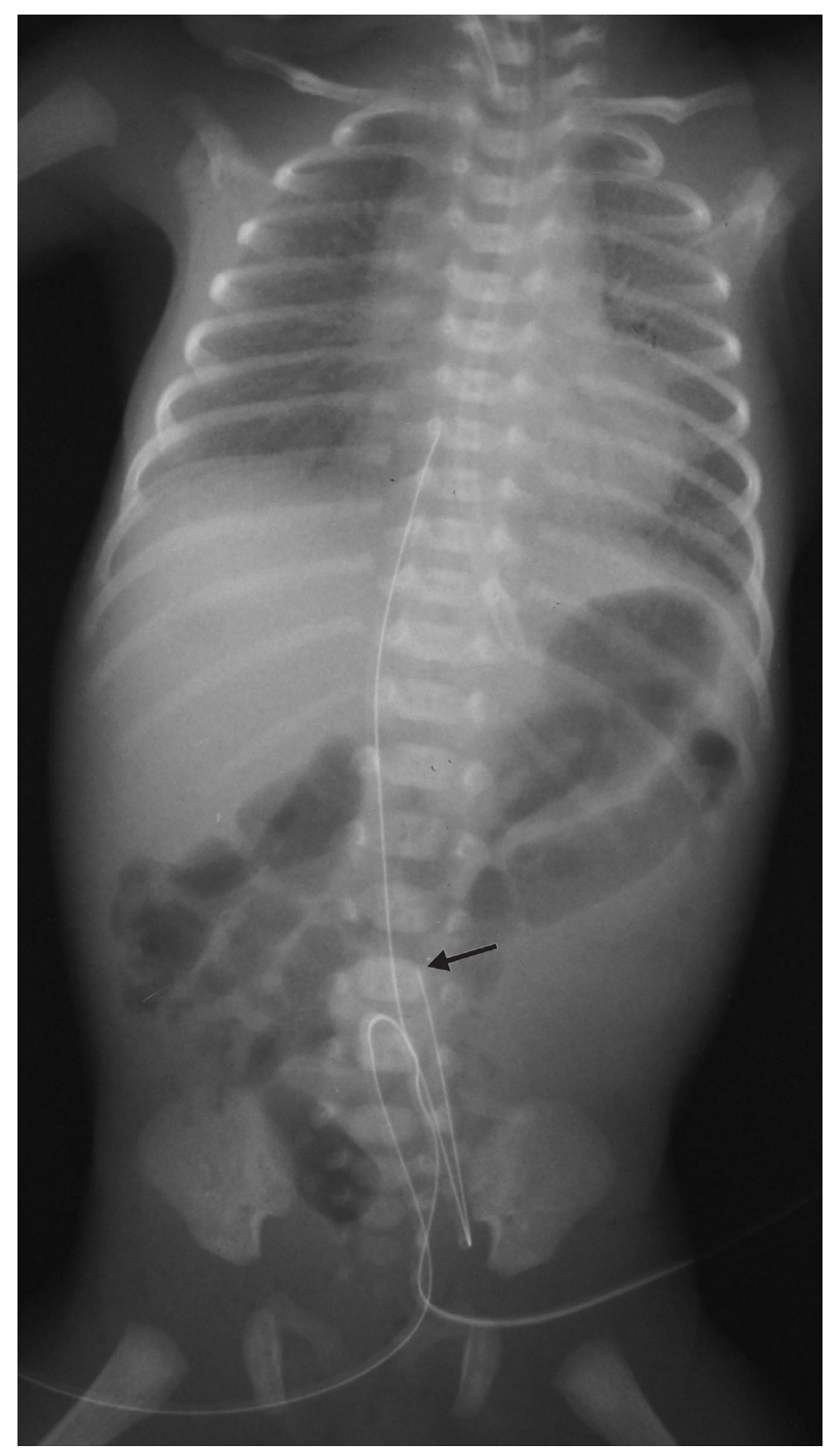

Figura 2. Radiografia de tórax e abdome de recém-nascido com 48 horas de vida, idade gestacional de 27 semanas e peso ao nascimento de $810 \mathrm{~g}$, apresentando doença da membrana hialina e submetido a cateterismo umbilical arterial e venoso. As extremidades distais dos cateteres são visíveis respectivamente ao nível do corpo vertebral de L4 (cateter umbilical arterial) em localização anatômica da aorta abdominal, logo acima da bifurcação das ilíacas (localização baixa) e à direita do corpo vertebral de T7 (cateter umbilical venoso), em localização anatômica do átrio direito (necessidade de reposicionamento). A sonda gástrica é visível em topografia do estômago. O recém-nascido está discretamente obliquado para a direita, mas é possível avaliar adequadamente os cateteres umbilicais, assim como o padrão pulmonar reticulogranular difuso com broncogramas aéreos periféricos, característico da doença da membrana hialina.

Fonte original: autora Beatriz Regina Alvares, publicada anteriormente em Alvares BR, Pereira ICMR, Araujo Neto SA, Skuma ETI. Achados normais no exame radiológico de tórax do recém-nascido. Radiol Bras 2006; 39(6):435-40. do átrio direito, devendo também ser reposicionado [3] (Figura 5). Quando a extremidade do cateter umbilical venoso estiver em topografia mais distante, ele pode estar localizado na veia cava superior, veia jugular, passar para o átrio esquerdo através do forâmen oval ainda patente e, inclusive, situar-se em um dos ramos da veia pulmonar, podendo ocasionar complicações como edema ou hemorragia pulmonar [55].

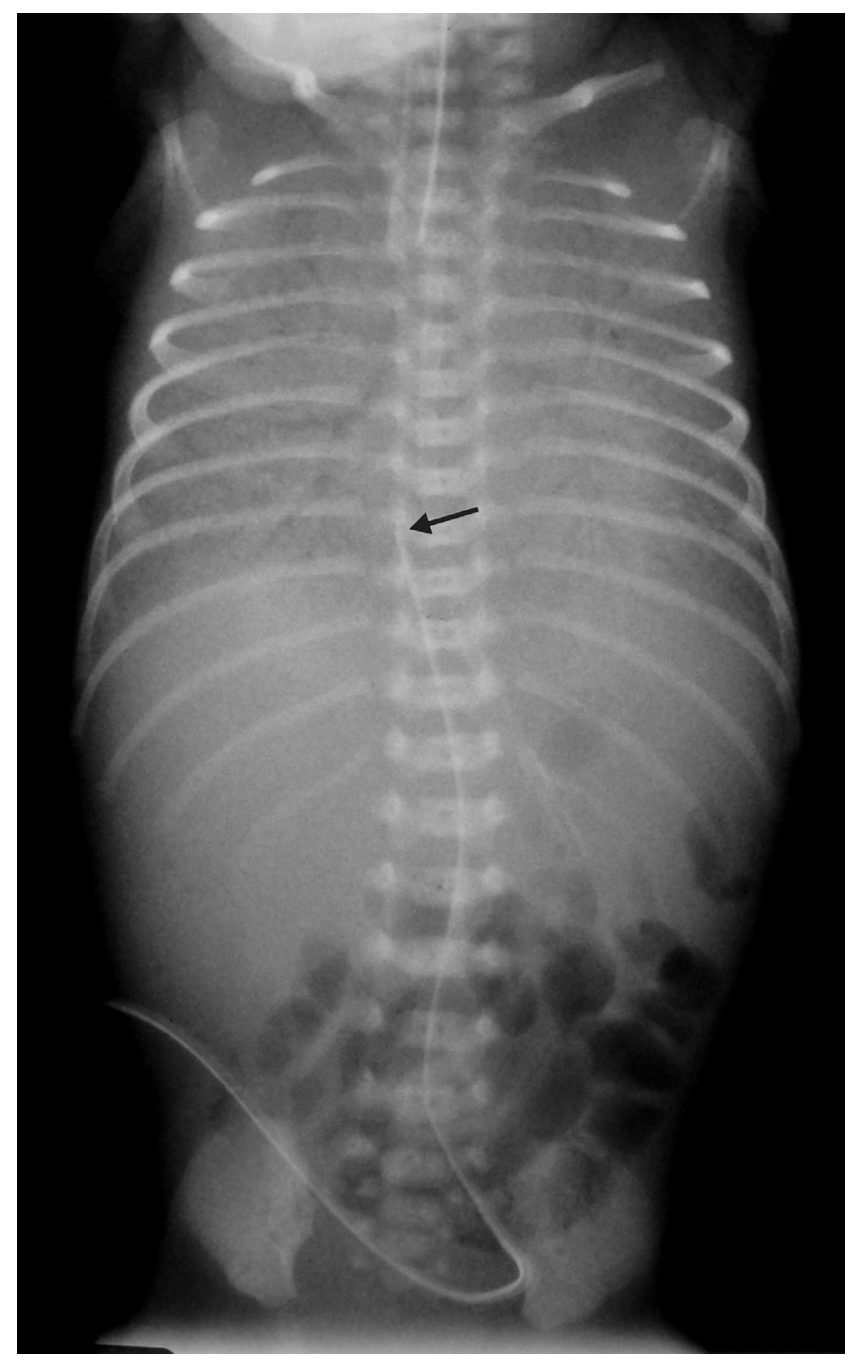

Figura 3. Radiografia de tórax e abdome de recém-nascido com 3 horas de vida, idade gestacional de 27 semanas, peso de nascimento de $920 \mathrm{~g}$, com doença da membrana hialina, submetido a cateterismo umbilical venoso. A extremidade distal do cateter umbilical encontra-se à direita do corpo vertebral de T8, em topografia anatômica da veia cava inferior, próxima ao átrio direito. A cânula endotraqueal é visível sobre o corpo vertebral de T2, abaixo da localização adequada (corpo vertebral de T1), precisando ser reposicionada. O recém-nascido está discretamente obliquado para a esquerda, sem prejuízo da análise radiológica, podendo também ser bem evidenciado o padrão reticulogranular difuso com broncogramas aéreos periféricos, característico da doença da membrana hialina. 


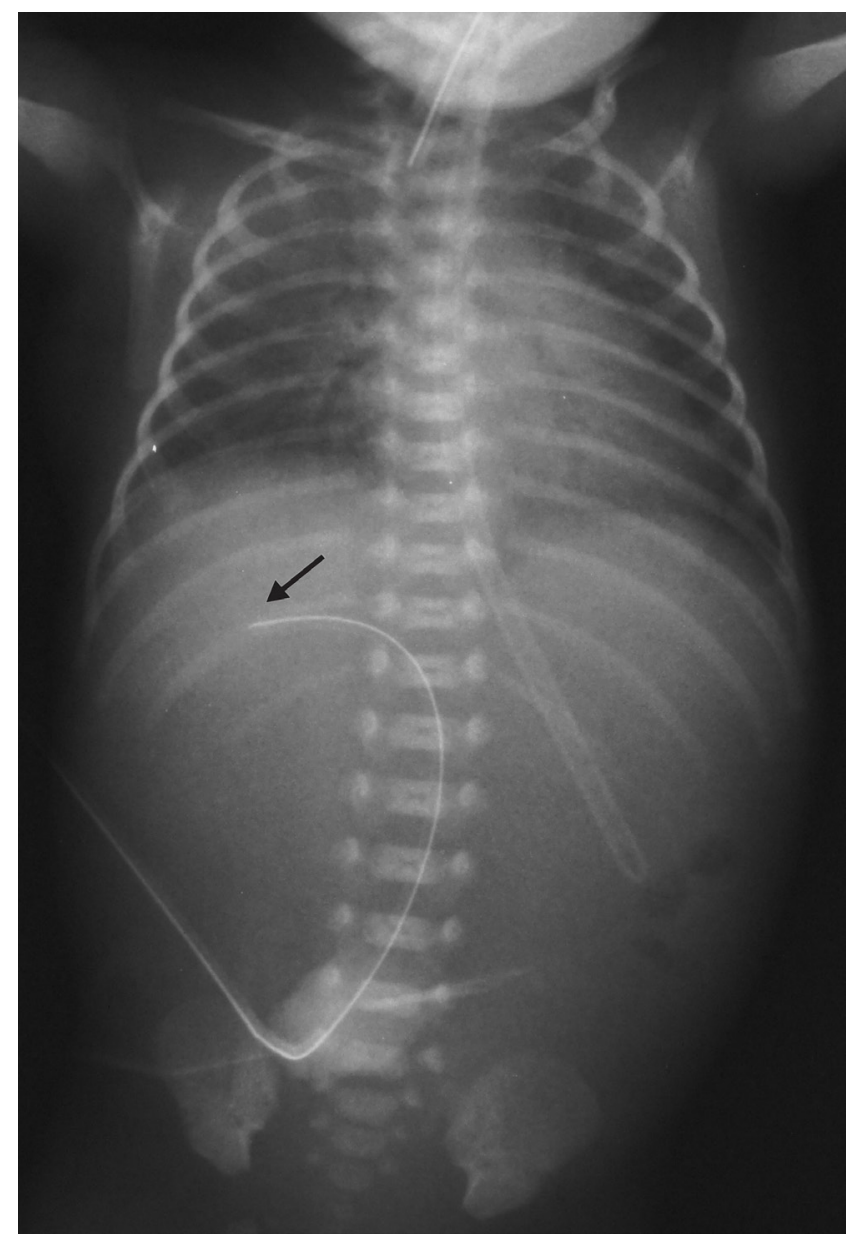

Figura 4. Radiografia de tórax e abdome de recém-nascido com 4 horas de vida, idade gestacional de 26 semanas, peso de nascimento de $765 \mathrm{~g}$, com insuficiência respiratória e submetido a cateterismo umbilical venoso. A extremidade distal do cateter umbilical encontra-se direcionada sobre a imagem hepática em topografia anatômica do ramo direito da veia porta, devendo o cateter ser removido. A extremidade da cânula endotraqueal é visível entre os corpos vertebrais de T1-T2, um pouco abaixo da localização adequada (corpo vertebral de T1) sendo desejável o seu reposicionamento. A sonda gástrica também está em localização muito baixa, devendo ser realocada. O recém-nascido está obliquado para a direita, porém é possível avaliar adequadamente os cateteres umbilicais, cânula endotraqueal e sonda gástrica, motivos da realização do exame radiológico.

\section{CONCLUSÕES}

O cateterismo umbilical representa um importante acesso à corrente sanguínea do recém-nascido, porém o procedimento não está isento de complicações, principalmente relacionadas ao mau posicionamento do cateter, além do seu tempo de permanência e características inerentes ao recém-nascido e à unidade hospitalar. O diagnóstico por imagem desempenha um papel fundamental na avaliação correta

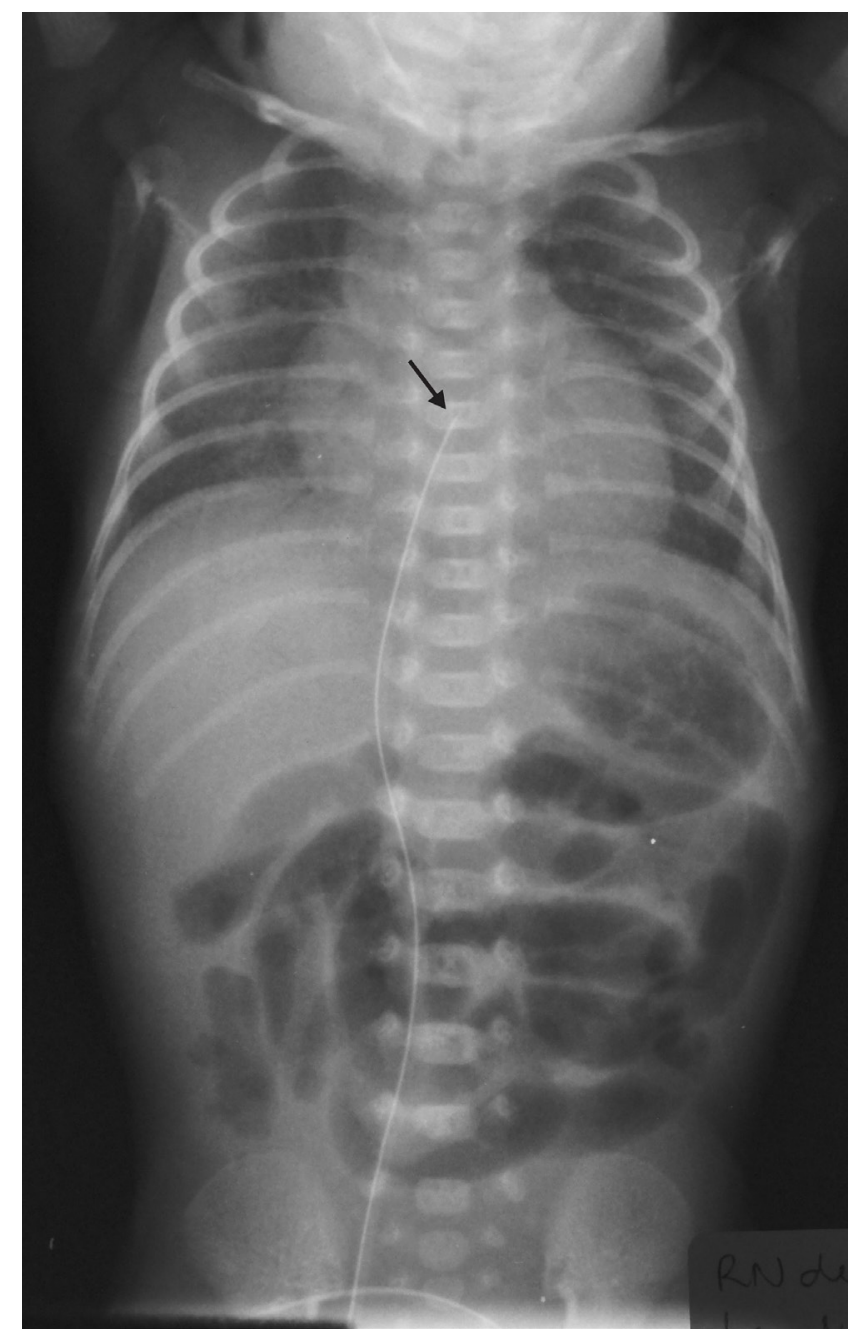

Figura 5. Radiografia de tórax e abdome de recém-nascido com 2 horas de vida, idade gestacional de 27 semanas, peso de nascimento de $900 \mathrm{~g}$, com doença da membrana hialina, submetido a cateterismo umbilical venoso. A extremidade distal do cateter umbilical encontra-se localizada ao nível do corpo vertebral de T6 em topografia de átrio direito, devendo ser reposicionado. A cabeça do recém-nascido encontra-se sobreposta ao terço superior do tórax, sem prejuízo para a avaliação radiológica do cateter umbilical venoso.

da localização do cateter, possibilitando, nos casos inadequados, a conduta imediata para prevenção de complicações. Apesar de a ultrassonografia apresentar algumas vantagens na sua utilização, a radiografia de tórax e abdome continua sendo o exame mais disponível e usado para garantir a correta localização dos cateteres umbilicais em recémnascidos. Existem várias fórmulas e referências anatômicas que permitem essa avaliação, as quais foram abordadas nesta revisão. 


\section{REFERÊNCIAS}

1. Brasil. Ministério da Saude. Atenção à saúde do recém-nascido: guia para profissionais de saúde. Intervenções comuns, icterícia e infecções. Brasília (DF); 2011.

2. Bradshaw WT, Furdon AS. A nurse's guide to early detection of umbilical venous catheter complications in infants. Ad Neonatal Care. 2006;6:127-38. http://dx.doi.org/10.1016/j.adnc.2006.03.002

3. Figueiredo Junior I, Lima GM. O conhecimento de neonatologistas relativo a técnicas de posicionamento de cateteres umbilicais. Pediatria (São Paulo). 2004;26(2):85-9.

4. Verheij GH, te Pas AB, Smits-Wintjens VE, Šràmek A, Walther FJ, Lopriore E. Revised formula to determine the insertion length of umbilical vein catheters. Eur J Pediatr. 2013;172(8):1011-5. http://dx.doi.org/10.1007/s00431-013-1981-Z

5. Butler-O'Hara M, Buzzard CJ, Reubens L, McDermott MP, DiGrazio W, D'Angio CT. A randomized trial comparing long-term and shortterm use of umbilical venous catheters in premature infants with birth weights of less than 1251 grams. Pediatrics. 2006;118:e25-35. http:// dx.doi.org/10.1542/peds.2005-1880

6. MacDonald MG, Mullett Md, Seshia MMK. Avery’s Neonatology: pathophysiology \& management of the newborn. 6th ed. Philadelphia: Lippincott Williams \& Wilkins; 2005.

7. Hermansen MC, Hermansen MG. Intravascular catheter complications in the neonatal intensive care unit. Clin Perinatol. 2005;32(1): 141-56. http://dx.doi.org/10.1016/j.clp.2004.11.005

8. Yiğiter M, Arda IS, Hiçsönmez A. Hepatic laceration because of malpositioning of the umbilical vein catheter: case report and literature review. J Pediatr Surg. 2008;43(5):E39-41. http://dx.doi.org/10.1016/j.jpedsurg.2008.01.018

9. Yumani DF, van den Dungen FA, van Weissenbruch MM. Incidence and risk factors for catheter-associated bloodstream infections in neonatal intensive care. Acta Paediatr. 2013;102(7):e293-8. http://dx.doi.org/10.1111/apa.12256

10. Beluffi G, Perotti G. Where has the umbilical catheter gone? An unusual position. Pediatr Radiol. 2007;37(4):403. http://dx.doi.org/10.1007/ s00247-006-0377-x

11. Eifinger F, Brisken K, Roth B, Koebke J. Topographical anatomy of central venous system in extremely low-birth weight neonates less than 1000 grams and the effect of central venous catheter placement. Clin Anat. 2011;24(6):711-6. http://dx.doi.org/10.1002/ca.21204

12. Grupo de Hospitales Castrillo. Studio prospectivo sobre el empleo de cateteres umbilicales em el récien nascido. An Esp Pediatr. 2000;53:470-78. http://dx.doi.org/10.1016/S1695-4033(00)78631-0

13. Nemri AMA, Inacio LC, Zamil FAA, Jarallah ASA. Rare but fatal complication of umbilical venous catheterization. Congenit Heart Dis 2006;1(4):180-3. http://dx.doi.org/10.1111/j.1747-0803.2006.00031.x

14. Greenberg M, Movahed H, Peterson B, Bejar R. Placement of umbilical venous catheters with use of bedside real-time ultrasonography. J Pediatr. 1995;126(4):633-5. http://dx.doi.org/10.1016/S0022-3476(95)70366-7

15. Lussky RC. Radiology casebook. Unusual misplacement sites for central venous catheters: three case reports. J Perinatol. 2000;20(8): 562-4. http://dx.doi.org/10.1038/sj.jp.7200462

16. Hogan MJ. Neonatal vascular catheters and their complications. Radiol Clin North Am. 1999;37(6):1109-25. http://dx.doi.org/10.1016/ S0033-8389(05)70252-9

17. Campbel RE. Roentgenologic features of umbilical vascular catheterization in the newborn. Am J Roentgenol Radium Ther Nucl Med. 1971;112(1):68-76. http://dx.doi.org/10.2214/ajr.112.1.68

18. Vali P, Fleming SE, Kim JH. Determination of umbilical catheter placement using anatomic landmarks. Neonatology. 2010;98(4):381-6. http://dx.doi.org/10.1159/000316918

19. Wright IMR, Owers M, Wagner M. The umbilical arterial catheter: a formula for improved positioning in the very low birth weight infant. Pediatr Crit Care Med. 2008;9(5):498-501. http://dx.doi.org/10.1097/PCC.0b013e318172d48d

20. Shukla H, Ferrara A. Rapid estimation of insertional length of umbilical catheters in newborns. Am J Dis Child. 1986;140:786-8. http:// dx.doi.org/10.1001/archpedi.1986.02140220068034

21. Dunn PM. Localization of the umbilical catheter by post-mortem measurement. Arch Dis Child. 1966;41(215):69-75. http://dx.doi. org/10.1136/adc.41.215.69

22. Slovis TL, editor. Caffey's Pediatric Diagnostic Imaging. 11th ed. Pennsylvania: Mosby Elsevier; 2008.

23. Furdon SA, Horgan MJ, Bradshaw WT, Clark DA. Nurses' guide to early detection of umbilical arterial catheter complications in infants. Adv Neonatal Care. 2006;6(5):242-60. http://dx.doi.org/10.1016/j.adnc.2006.06.001

24. Imamura T, Momoi N, Go H, Ogasawara K, Kanai Y, Sato M, Goto A, Hosoya M. Evaluation of arterial catheter management in very preterm neonates: peripheral artery versus umbilical artery. Fukushima J Med Sci. 2012;58(1):1-8. http://dx.doi.org/10.5387/fms.58.1

25. Coleman MM, Spear ML, Finkelstein M, Leef KH, Pearlman SA, Chien C, Taylor SM, McKenzie SE. Short term use of umbilical artery catheters may not be associated with increased risk of thrombosis. Pediatrics. 2004;113(4):770-4. http://dx.doi.org/10.1542/peds.113.4.770

26. Demirel N, Aydin M, Zenciroglu A, Bas AY, Yarali N, Okumus N, Cinar G, Ipek S. Neonatal thrombo-embolism: risk factors, clinical features and outcome. Ann Trop Paediatr. 2009;29(4):271-9. http://dx.doi.org/10.1179/027249309X12547917868961

27. Narang S, Roy J, Stevens TP, Butler-O'Hara M, Mullen CA, D’Angio CT. Risk factors for umbilical venous catheter-associated thrombosis in very low birth weight infants. Pediatr Blood Cancer. 2009;52(1):75-9. http://dx.doi.org/10.1002/pbc.21714

28. Boo NY, Wong NC, Zulkifli SS, Lye MS. Risk factors associated with umbilical vascular catheter-associated thrombosis in newborn infants. J Paediatr Child Health. 1999;35(5):460-5. http://dx.doi.org/10.1046/j.1440-1754.1999.355392.x 
29. Kim JH, Lee YS, Kim SH Lee SK, Lim MK, Kim HS. Does umbilical vein catheterization lead to portal venous thrombosis? Prospective US evaluation in 100 neonates. Radiology. 2001;219(3):645-50. http://dx.doi.org/10.1148/radiology.219.3.r01jn17645

30. Morag I, Epelman M, Daneman A, Moineddin R, Parvez B, Shechter T, Hellmann J. Portal vein thrombosis in the neonate: risk factors, course, and outcome. J Pediatr. 2006;148(6):735-9. http://dx.doi.org/10.1016/j.jpeds.2006.01.051

31. Kirpalani H, Mernagh J, Epelman M. Imaging of the Newborn. Cambridge: Cambridge University Press; 2011. http:/dx.doi.org/10.1017/ CBO9780511978074

32. Schlapbach LJ, Pfammatter JP, Nelle M, McDougal FJ. Cardiomegaly in a premature neonate after venous umbilical catheterisation. Eur J Pediatr. 2009;168(1):107-9. http://dx.doi.org/10.1007/s00431-008-0704-3

33. Onal EE, Saygili A, Koç E, Türkyilmaz C, Okumus N, Atalay Y. Cardiac tamponade in a newborn because of umbilical venous catheterization: is correct position safe? Paediatr Anaesth. 2004;14(11):953-6. http://dx.doi.org/10.1111/j.1460-9592.2004.01385.x

34. Verheij G, Smits-Wintjens V, Rozendaal L, Blom N, Walther F, Lopriore E. Cardiac arrhythmias associated with umbilical venous catheterisation in neonates. BMJ Case Rep. 2009;2009. http://dx.doi.org/10.1136/bcr.04.2009.1778

35. Remington JS, Klein JO, Wilson CB, Nizet V, Maldonado YA, editors. Infectious Diseases of the Fetus and Newborn Infant. 7th ed Pennsylvania: Elsevier Saunders; 2011.

36. Chung DH, Ziegler MM. Central venous catheter access. Nutrition. 1998;14(1):119-23. http://dx.doi.org/10.1016/S0899-9007(97)00228-1

37. Yalaz M, Altun-Köroğlu O, Ulusoy B, Yildiz B, Akisu M, Vardar F, Ozinel MA, Kültürsay N. Evaluation of device-associated infections in a neonatal intensive care unit. Turk J Pediatr. 2012;54(2):128-35.

38. Bothur-Nowacka J, Czech-Kowalska J, Gruszfeld D, Nowakowska-Rysz M, Kościesza A, Polnik D, Dobrzańska A. Complications of umbilical vein catherisation. Case Report. Pol J Radiol. 2011;76(3):70-3.

39. Costa S, Carolis MPD, Savarese I, Manzoni C, Lacerenza S, Romagnoli C. An unusual complication of umbilical catheterisation. Eur J Pediatr. 2008;167(12):1467-9. http://dx.doi.org/10.1007/s00431-008-0691-4

40. Mogbo KI, Wang DC. Biliary venous fistula from umbilical catheter placement. Pediatr Radiol. 1997;27(4):333-5. http://dx.doi.org/10.1007/ s002470050143

41. Alvares BR, Stopiglia MCS, Mezzacappa MA. Presence of air in the hepatic portal system in association with umbilical venous catheter malposition. Radiol Bras. 2014;47(1):49-50. http://dx.doi.org/10.1590/S0100-39842014000100015

42. Houston AB, Garg AK, Maclaurin JC, Mackenzie JR. Ultrasound positioning of umbilical arterial catheters. Lancet. 1982;2(8301): 759. http://dx.doi.org/10.1016/S0140-6736(82)90934-5

43. Garg AK, Houston AB, Laing JM, Mackenzie JR. Positioning of umbilical arterial catheters with ultrasound. Arch Dis Child. 1983;58(12):1017-8. http://dx.doi.org/10.1136/adc.58.12.1017

44. Michel F, Brevaut-Malaty V, Pasquali R, Thomachot L, Vialet R, Hassid S, Nicaise C, Martin C, Panuel M. Comparison of ultrasound and X-ray in determining the position of umbilical venous catheters. Resuscitation. 2012;83(6):705-9. http://dx.doi.org/10.1016/j. resuscitation.2011.11.026

45. Simanovsky N, Ofek-Shlomai N, Rozovsky K, Ergaz-Shaltiel Z, Hiller N, Bar-Oz B. Umbilical venous catheter position: evaluation by ultrasound. Eur Radiol. 2011;21(9):1882-6. http://dx.doi.org/10.1007/s00330-011-2129-z

46. Bayhan C, Takci S, Çiftçi TT, Yurdakök M. Sterile hepatic abscess due to umbilical venous catheterization. Turk J Pedriatr. 2012; 54:671-3.

47. Vali P, Fleming SE, Kim JH. Determination of umbilical catheter placement using anatomic landmarks. Neonatology. 2010;98(4):381-6. http://dx.doi.org/10.1159/000316918

48. Schelesinger AE, Braverman RM, DiPietro MA. Neonates and umbilical venous catheters: normal appearance, anomalous positions, complications, and potential aid to diagnosis. AJR Am J Roentgenol. 2003;180:1147-53. http://dx.doi.org/10.2214/ajr.180.4.1801147

49. Narla LD, Hom M, Lofland GK. Evaluation of umbilical catheter and tube placement in premature infants. Radiographics. 1991;11(5): 849-63. http://dx.doi.org/10.1148/radiographics.11.5.1947320

50. Radiology in the nursery - indications, positioning and safety. [Internet]. Los Angeles; 2013 [cited 2013 June]. Available from: http:// paclac.org/guidelines-care/\#neonatal

51. Peck DR. Lowman RM. Roentgen aspects of umbilical vascular catheterization in the newborn. The problems of catheter placement. Radiology. 1967;89(5):874-7. http://dx.doi.org/10.1148/89.5.874

52. Baker DH, Berdon WE, James LS. Proper localization of umbilical arterial and venous catheters by lateral roentgenograms. Pediatrics. $1969 ; 43(1): 142-3$

53. Alvares BR, Pereira ICMR, Araujo Neto SA, Skuma ETI. Achados normais no exame radiológico de tórax do recém-nascido. Radiol Bras. 2006;39(6):435-440. http://dx.doi.org/10.1590/S0100-39842006000600012

54. Campbel RE. Roentgenologic features of umbilical vascular catheterization in the newborn. Am J Roentgenol Radium Ther Nucl Med. 1971;112(1):68-76. http://dx.doi.org/10.2214/ajr.112.1.68

55. Seguin J, Fletcher MA, Landers S. Umbilical venous catheterizations: audit by the study group for complications perinatal care. Am J Perinatol. 1994; 11:67-70. http://dx.doi.org/10.1055/s-2007-994540

56. Oestreich AE. Umbilical vein catheterization-appropriate and inappropriate placement. Pediatr Radiol. 2010;40(12):1941-9. http://dx.doi. org/10.1007/s00247-010-1840-2 